\title{
Intervenções de profissionais de enfermagem para a assistência espiritual: uma revisão narrativa
}

Interventions by nursing professionals for spiritual assistance: notes for a perennial debate

Intervenciones de profesionales de enfermería para la asistencia espiritual: notas para un debate perennial

Sonia Hutul Silva ${ }^{1}$, Beatriz Maria dos Santos Santiago Ribeiro ${ }^{2 *}$, Fabio Scorsolini-Comin², Carlos Takeo Okamura ${ }^{1}$, Eleine Aparecida Penha Martins ${ }^{1}$.

\section{RESUMO}

Objetivo: Refletir, a partir de uma narrativa da literatura científica, sobre as intervenções de profissionais de enfermagem para a assistência espiritual. Revisão bibliográfica: Partiu da atual Classificação das Intervenções de Enfermagem, que menciona 56 ações relacionadas ao cuidado religioso-espiritual, bem como do diálogo entre essas recomendações e os estudos consolidados na área de religiosidade/espiritualidade e saúde. Destaca-se que a assistência de cunho religioso-espiritual ofertada pelo enfermeiro deve respeitar dilemas éticos, crenças religiosas e cuidados de saúde, bem como a própria opção do paciente ou de sua família de não incluírem esses elementos no cuidado. O profissional, dessa forma, deve estar conectado com essas necessidades, abrindo-se à escuta do paciente e de seus familiares em relação a essa dimensão. Considerações finais: Ao se apropriar da temática da religiosidade e da espiritualidade o enfermeiro conhece subsídios e aportes teóricos para nortear a aplicação de intervenções espirituais no processo de tomada de decisão para a condução da assistência.

Palavras-Chave: Espiritualidade, Religião, Enfermagem.

\section{ABSTRACT}

Objective: To reflect, from a narrative of the scientific literature, on the interventions of nursing professionals for spiritual assistance. Literature review: Based on the current Classification of Nursing Interventions, which mentions 56 actions related to religious-spiritual care, as well as the dialogue between these recommendations and the consolidated studies in the area of religiosity / spirituality and health. It is noteworthy that the assistance of a religious-spiritual nature offered by the nurse must respect ethical dilemmas, religious beliefs and health care, as well as the patient's or family's own option of not including these elements in care. The professional, therefore, must be connected with these needs, opening up to listening to the patient and his family in relation to this dimension. Final considerations: When appropriating the theme of religiosity and spirituality, the nurse knows subsidies and theoretical contributions to guide the application of spiritual interventions in the decisionmaking process for conducting care.

Key words: Spirituality, Religion, Nursing.

\section{RESUMEN}

Objetivo: Reflexionar, a partir de una narrativa de la literatura científica, sobre las intervenciones de profesionales de enfermería para asistencia espiritual. Revisión bibliográfica: Basada en la Clasificación actual de intervenciones de enfermería, que menciona 56 acciones relacionadas con el cuidado religiosoespiritual, así como el diálogo entre estas recomendaciones y los estudios consolidados en el área de

1 Universidade Estadual de Londrina (UEL), Londrina - PR.

2 Universidade de São Paulo (USP), Ribeirão Preto - SP.

*E-mail: beatrizsantiag01994@hotmail.com

SUBMETIDO EM: 5/2020 
religiosidad / espiritualidad y salud. Es de destacar que la asistencia de carácter religioso-espiritual ofrecida por la enfermera debe respetar los dilemas éticos, las creencias religiosas y la atención médica, así como la opción del paciente o la familia de no incluir estos elementos en la atención. El profesional, por lo tanto, debe estar conectado con estas necesidades, abriéndose a escuchar al paciente y su familia en relación con esta dimensión. Consideraciones finales: Al apropiarse del tema de religiosidad y espiritualidad, la enfermera conoce subsidios y contribuciones teóricas para guiar la aplicación de intervenciones espirituales en el proceso de toma de decisiones para llevar a cabo la atención.

Palabras clave: Espiritualidad, Religión, Enfermería.

\section{INTRODUÇÃO}

O conceito de religiosidade/espiritualidade $(R / E)$ possui inequívoca implicação no campo da enfermagem. Por essa razão, tal noção deve ser estudada e considerada como componente relevante da práxis de Enfermagem, em consonância com as orientações da Organização Mundial da Saúde (OMS), que incluem o cuidado religioso-espiritual no rol de procedimentos que devem ser corporificados na atenção em saúde (MENDONÇA AG, et al., 2018).

Mesmo com as dificuldades inerentes à conceituação dessa dimensão, o que envolve noções como as de religião, de religiosidade e de espiritualidade, estudos recentes mostram a importância da inclusão desses elementos no cuidado a pacientes, relatando resultados benéficos no processo de enfrentamento a diversos tipos de patologia (SOUSA FFPR, et al., 2017; FREIRE MEM, et al., 2017).

Uma farta literatura a respeito dos desfechos positivos em saúde associados à inclusão do cuidado religioso-espiritual tem sido recuperada e divulgada na contemporaneidade, promovendo uma atenção cada vez mais consistente em relação a essas evidências (KOENIG HG, 2007).

Em que pesem as aproximações e os distanciamentos entre as noções de religiosidade e de espiritualidade, Almeida AM e Lucchetti G (2016) utilizam a definição de espiritualidade como sendo a relação com o sagrado (Deus, poder superior). O espírito refere-se a uma dimensão não material (Deus ou deuses, almas, anjos, demônios).

A religião se configura como um sistema organizado de crenças e práticas que facilitam a proximidade com o transcendente, são instituições organizadas. Os autores Caldeira S, et al. (2016) afirmam que espiritualidade está presente em crentes e não crentes, sendo uma dimensão humana dinâmica, integrativa e multidimensional. Já os autores Neely D e Minford EJ (2008) a conceituam como uma consciência do significado final e propósito da vida, com uma crença em um poder superior no universo, podendo ou não estar associado a uma organização religiosa.

Ainda que se possa considerar epistemologicamente as diferenças entre os conceitos supracitados, neste estudo adota-se a nomenclatura combinada religiosidade/espiritualidade $(R / E)$, conforme sugerido pela literatura no campo da. A nomenclatura combinada tem o objetivo de salientar as aproximações entre esses vértices ao pensar o cuidado, priorizando a discussão acerca de como incluir, de fato, esse componente na atenção em saúde ou como os profissionais podem se posicionar diante dos protocolos que recomendam tais práticas.

Assim, os efeitos desses termos nos desfechos em saúde seriam melhores investigados com a padronização da nomenclatura combinada, permitindo a inclusão de experiências e reflexões diversas que podem fazer parte do rol de cuidados religiosos-espirituais, sobretudo em um contexto como o brasileiro, em que esses elementos se mostram, muitas vezes, sobrepostos, o que não diminui a importância do fenômeno (CUNHA VF e SCORSOLINI-COMIN F, 2019).

Mas de que modo a R/E tem se mostrado presente no campo da Enfermagem? Em que pesem as diversas reflexões teóricas presentes na literatura científica, as lacunas práticas acerca de como incorporar essa dimensão na atuação em saúde mostram-se evidentes. 
A Classificação das Intervenções de Enfermagem (NIC) traz apontamentos importantes no sentido de, efetivamente, corporificar as recomendações da OMS na oferta de um cuidado que considera a dimensão religiosa-espiritual do paciente.

Desse modo, a NIC é um ponto estratégico e aprofundado no chamado aconselhamento espiritual, apoiando a expressão das necessidades religiosas e espirituais, os sentimentos dos envolvidos, a resolução dos problemas, o apoio no enfrentamento do processo de saúde-doença e as relações interpessoais (BULECHEK GM, et al., 2016).

Assim, é mister que entre as NIC possam ser incluídas as discussões acerca da R/E no cuidado em saúde, priorizando uma atenção sistêmica que esteja em consonância com os princípios da Organização Mundial da Saúde e das melhores práticas em saúde vigentes na contemporaneidade. Além disso, trata-se de um processo que pode contribuir não apenas com a ampliação das competências dos profissionais de enfermagem, mas também disparar reflexões por parte das universidades e centros de formação para que, de fato, incluam em seus componentes curriculares uma discussão séria e embasada não somente acerca da R/E, mas de como manejá-la na promoção do cuidado.

Tal desafio envolve questionamentos que, por vezes, cotejam as NIC diante das recomendações para a incorporação da R/E na atenção em saúde. Como a R/E poderia dialogar com as NIC de maneira integrada?

Apesar do reconhecimento da necessidade de cada vez mais trazer a R/E para a linha de cuidado, há uma escassez de estudos que orientem o escopo de intervenções espirituais ou que estejam orientadas para tal dimensão. Devido a essa lacuna no campo da prática em Enfermagem e a necessidade de suporte espiritual nas instituições de saúde (MENDONÇA AG, et al., 2018). Sendo assim, objetivou-se refletir através de uma revisão narrativa sobre as intervenções de profissionais de enfermagem para a assistência espiritual.

\section{REVISÃO BIBLIOGRÁFICA}

Um primeiro aspecto a ser destacado é que, para haver a incorporação da R/E no cuidado em saúde, o profissional de Enfermagem não precisa ser religioso ou um praticante de qualquer religião. $O$ que é necessário é que esse profissional esteja atento à causa do sofrimento, que pode envolver o mundo religiosoespiritual do paciente. É importante que esse profissional possa verificar se a oferta de escuta ou de uma prática que considere a dimensão religiosa-espiritual do paciente é algo desejado por este ou mesmo por sua família, qualquer que seja o tipo de adoecimento experienciado, em todas as etapas do processo de cuidar. A assistência espiritual respeita dilemas éticos, crenças religiosas e cuidados de saúde. O profissional, dessa forma, deve estar conectado com essas necessidades, abrindo-se à escuta do paciente e de seus familiares em relação a essa dimensão (SIMÕES ND, et al., 2018).

Ao se utilizar das NIC no processo de trabalho de Enfermagem, além de ser importante ferramenta no processo pedagógico de capacitação de enfermeiros para verificar a necessidade do paciente e intervir adequadamente em cada caso, lacunas dos conhecimentos podem ser identificadas e utilizadas para apoiar futuras pesquisas. É recomendável a utilização de resultados da Classificação dos resultados de enfermagem (NOC), intervenções da NIC e diagnósticos da NANDA-I, pois são importantes ferramentas para tomada de decisões clínicas pelo enfermeiro e desenvolvimento de conhecimento científico (BULECHEK GM, et al., 2016). No entanto, este tema sugere outros estudos, embora o tema da R/E esteja contemplado nestes importantes instrumentos.

As ações classificadas na NIC são um ponto de partida para mais esta contribuição prática da R/E na prática da Enfermagem pois, como descrito na assistência direta ao paciente, o "toque das mãos" e ações de aconselhamento feitas pelo enfermeiro como intervenção assistencial, respaldado em um diagnóstico de Enfermagem, baseado em raciocínio científico em benefício ao paciente.

Fatores como diagnóstico de Enfermagem, resultados desejados, viabilidade da intervenção, aceitação do paciente e competência técnica do enfermeiro são premissas para a seleção da NIC, dando a está um caráter personalizado, tanto para enfermeiro que executa como para o paciente que recebe (BULECHEK GM, et al., 2016). A colaboração multiprofissional deve ser organizada pelo enfermeiro quando necessário. 
Dentre as 542 intervenções de Enfermagem estabelecidas na quinta edição da NIC, encontram-se organizadas em sete domínios e 30 classes (BULECHEK GM, et al., 2016). Destacamos para este estudo a presença do termo espiritual, que aparece 56 vezes. Por meio do apoio espiritual, a assistência de enfermagem colabora para o equilíbrio e a conexão do paciente com um plano superior. Pode também ofertar e facilitar o apoio espiritual ao paciente e seus familiares a partir do contato com o religioso, conforme apropriado, admitindo o paciente sem julgamentos aos seus antecedentes espirituais e culturais.

O profissional de enfermagem também pode encorajar o paciente com o apoio em recursos espirituais, se desejado, para expandir a espiritualidade do mesmo. Assim, nota-se que existe um rol de competências bastante amplo em relação ao apoio espiritual que pode ser ofertado pelo enfermeiro (BULECHEK GM, et al., 2016).

A NIC relaciona-se à facilitação do crescimento espiritual para identificar, fazer conexão e chamar a fonte de sentido com a finalidade de promover conforto, fortalecimento e esperança na vida do paciente. Para esta intervenção, sugere-se que o enfermeiro apresente-se com carinho, possibilitando o diálogo sobre preocupações espirituais, estímulo à autodescoberta, dando suporte com orações individuais ou em grupo, participação de serviços de devoção, celebrações e rituais espirituais, bem como adequação de ambiente para prática da meditação, crenças à cura corporal, mental e espiritual.

Também envolve a participação ou a oferta de grupos de apoio e programas de base espiritual que podem proporcionar cuidados religiosos e outros adicionais para conectar corpo, mente e espírito (BULECHEK GM, et al., 2016).

Outro ponto importante apresentado na NIC é a dimensão da R/E que recomenda a Presença da Família. Desse modo, consideram-se as necessidades de apoio emocional, físico, psicossocial e espiritual tanto da família quanto do paciente. O apoio familiar - NIC tem como definição a promoção dos valores, interesses e metas da família. Tem como atividade identificar a natureza do apoio espiritual para a família, oferecendo recursos espirituais, orientando sistemas de apoio espirituais.

Também nos deparamos com a NIC Estimulação da Imaginação, que indica a atividade de individualizar as imagens escolhidas considerando crenças espirituais ou religiosas. Em Assistência ao Morrer, orienta-se que enfermeiro deve facilitar a obtenção de apoio espiritual ao paciente e às famílias (BULECHEK GM, et al., 2016).

Ainda temos a NIC Terapia ocupacional, que prescreve assistência espiritual, e a NIC Facilitação do Processo de Perdão, auxiliando para que o enfermeiro possa colaborar para que o paciente venças seus anseios e bloqueios para a cura usando práticas espirituais através de orações, de louvor, rezas, discernimento, toque e visualização da cura e agradecimento. Obviamente que esses repertórios serão também direcionados pelas crenças, necessidades e também abertura por parte do paciente.

O termo espiritual ainda aparece na NIC Enfermagem de Cuidados Paliativos e NIC Enfermagem do Controle da Dor, Enfermagem Holística, Enfermagem na Dependência, Enfermagem Oncológica, Enfermagem Paroquial, com Apoio Espiritual e/ou Facilitação do Crescimento Espiritual. Estas intervenções necessitam de 16 a 30 minutos e o crescimento espiritual de 31 a 45 minutos (BULECHEK GM, et al., 2016).

O profissional de Enfermagem, na maioria das vezes, encontra-se com dificuldade em utilizar padrões de informações produzidos nas áreas de R/E. Ao longo de toda a sua formação, é orientado a manter a cientificidade, a zelar pela ética, buscando a humanização, o respeito em seu atendimento e a sensibilidade.

Embora todos esses elementos e recomendações estejam alinhados ao domínio da R/E, a incorporação desse aspecto no cuidar constitui, ao mesmo tempo, um desafio (MENDONÇA AG, et al., 2018), haja vista que ainda nos sustentamos em um paradigma positivista e dentro de um modelo biomédico que, apesar de ser criticado, ainda orienta o modo como os profissionais pensam o cuidado.

A Enfermagem vem buscando ao longo dos anos o entendimento sobre o que é R/E e a assistência espiritual, utilizando reflexões da ética, bioética, filosofia e mesmo da religião. Pode-se, assim, pressupor a necessidade de inclusão desta reflexão na prática da enfermagem que frequentemente se depara com o limite entre a vida e a morte, dor e sofrimento (PEDRÃO RB, BERESIN R, 2010). 
Assistir ao paciente em sua dor coloca o enfermeiro como participante daquele momento de vida ou de morte, refletindo também em seu estado emocional, psíquico e espiritual, mesmo que não perceba ou não se dê conta disso. A conexão espiritual com o paciente, por meio da meditação e da oração, por exemplo, possui potencial para o alívio de sintomas, desenvolvimento de uma atitude de esperança e abertura para fenômenos de fé que podem ser de difícil explicação pela medicina atual.

A intervenção de Enfermagem, nesse sentido, pode envolver orações, meditações e leituras de textos sagrados juntamente com os pacientes (MILLER AJ, 2018). Ressalta-se que o enfermeiro deve avaliar 0 posicionamento do enfermo no momento das intervenções médicas/de enfermagem, de modo que não se trata apenas de uma escolha ou decisão do profissional, devendo-se considerar os posicionamentos trazidos pelos pacientes, respeitando suas condições, desejos e repertórios (MENDONÇA AG, et al., 2018).

Entretanto, é fundamental que o enfermeiro esteja preparado para a abordagem espiritual, a fim de alcançar a sintonia necessária com o paciente, dando um salto no cuidado assistencial para uma relação transpessoal, caso seja essa a escolha da pessoa no itinerário do processo de saúde-doença.

Ouvir e estar atento aos sentidos físicos e espirituais do paciente proporcionará ao enfermeiro uma maior aproximação com a sua intimidade, melhorando a qualidade das relações assistenciais. Um exemplo é a prática do mindfulness, apontada na Teoria do Cuidado Humano, busca a consciência plena, tendo sido descrita em outros estudos a melhora de os estados de humor, redução de ansiedade e depressão.

A partir dessas práticas, pacientes e enfermeiros são envolvidos em estados de harmonia, pois a espiritualidade não é uma mão única para quem oferece, mas um dar e receber para ambos, terapeuta e paciente que serão alcançados em suas dores e sofrimentos (MENDONÇA AG, et al., 2018). As práticas meditativas em geral atuam reduzindo pensamentos e sentimentos negativos através da intervenção espiritual (CHEUNG KC, et al., 2017).

As rezas e orações também são intervenções de Enfermagem classificadas no NIC (BULECHEK GM, et al., 2016). Contudo, deve solicitar permissão para realizar tal intervenção de Enfermagem, avaliando opiniões do paciente e sua vivência espiritual (CAMPOS AF, RIBEIRO JP, 2017).

As orações ou preces intercessoras são súplicas feitas em intenção de ajuda de alguém, independentemente de crença, denominação religiosa ou mesmo não crentes. A palavra que vem do latim prexi, significa pedido, súplica. Sendo aceito pelo paciente, esta prática pode ser feita através de orações de intercessão por um grupo de pessoas ou uma única pessoa (CARVALHO CC, 2013).

Essas ações podem ser realizadas por meio de prece silenciosa, por exemplo, como forma de acolher o paciente em sua dor, colocando-se ao seu lado e em silêncio pedir por seu bem-estar. A intenção posta será percebida pelo paciente ao sentir interesse do enfermeiro pela sua comodidade (BULECHEK GM, et al., 2016)

A aplicação da prática espiritual causa promoção do bem-estar dos envolvidos (ROBITSCHEK C, HARDIN EE, 2017), sendo a espiritualidade uma dimensão da totalidade humana a ser explorada na relação fenomenológica existencial (BORIS GDJB, et al., 2017).

Nesse momento, o enfermeiro torna-se coautor no processo terapêutico espiritual (JANG JE, 2016). Utiliza-se de estratégias que reduzem a depressão e ansiedade, colaborando para a melhora do humor e da qualidade de vida do atendimento aos enfermos (CARLSON LE, et al., 2016).

Vale salientar que o uso de um modelo de aconselhamento multicultural no atendimento em saúde preconiza a cultura como parte essencial das intervenções espirituais (SCORSOLINI-COMIN F, 2015). A Enfermagem deve ter amplos conhecimentos em bioética e cultura religiosa para conduzir tais situações, pois pacientes podem escolher líderes espirituais da mesma orientação religiosa para receber orientações diretivas com intuito de amenizar o seu sofrimento.

Nesse sentindo, é parte do processo da integração do indivíduo com sua própria espiritualidade (MENDONÇA AG, et al., 2018). Os desafios para incorporar a R/E na prática da Enfermagem são vários, a começar pela forma com que o profissional acolhe a sua própria espiritualidade, a qual se desenvolve ao longo da vida e na reflexão do significado e razão de viver. 
Devido à diversidade de conceitos acerca da religiosidade e da espiritualidade, como apontado anteriormente, a Enfermagem precisa atentar-se às necessidades do paciente, assim como ter clareza de qual o significado ou entendimento do tema o assistido possui.

Somente no papel de escuta verdadeira, demonstrando empatia e possibilitando a interação eficaz é que o enfermeiro consegue de maneira satisfatória atender à demanda do paciente. Este é um desafio para ser trabalhado pelo enfermeiro, uma vez que também se trata de um ser humano que carrega consigo crenças, valores e significados, dentro de modelos culturais que nem sempre podemos conhecer com propriedade. Despir-se dos próprios conhecimentos e valores para ouvir e atender prontamente às necessidades espirituais de outra pessoa pode exigir um grande exercício do enfermeiro: "dar uma volta no mundo do paciente", sabendo que não haverá perdas ou danos nas suas próprias crenças ou convicções (SIMÕES ND, et al., 2018).

A equipe de Enfermagem deve ter preparo e competência técnica para acolher o sofrimento espiritual do paciente. Com isso, pode aumentar a confiança e fortalecer o elo entre equipe de Enfermagem, pacientes e familiares. As intervenções espirituais mencionadas no presente estudo podem contribuir, sobremaneira, com o enfermeiro durante sua prática profissional.

As intervenções apontadas na NIC apontam práticas possíveis de serem aplicadas durante a assistência de Enfermagem, necessitando, entretanto, de sensibilização do profissional enfermeiro. Dessa maneira, citamos, dentre várias, a criação de um ambiente que facilite a pratica religiosa, a possibilidade de encorajar a família sobre os aspectos positivos da expressão da esperança junto ao paciente, evitando mascarar a verdade e acolhendo com escuta ativa suas necessidades (BULECHEK GM, et al., 2016). Esse processo envolve, inequivocamente, a necessidade de a equipe de saúde estar aberta para esse tipo de abordagem e para a oferta dos cuidados espirituais ou relacionados a essa dimensão descritos na NIC. Embora essas orientações possam ser acessadas por todos os profissionais de Enfermagem, há que se reconhecer que, por diferentes motivos, esses profissionais podem ter mais ou menos disponibilidade para o cotejamento dessas ações.

Instrumentalizar essas ações a partir de uma reflexão institucional pode ser um caminho para que esses cuidados possam ser ampliados e, de fato, atingirem seus objetivos quanto aos desfechos em saúde. Obviamente que se trata de um processo complexo e que envolve, muitas vezes, a ruptura com modelos que ainda não incorporam devidamente a $R / E$ ao cuidado ou que a concebem em meio a incertezas e desconfianças. As NIC cumprem um papel importante nesse sentido, o que é enfatizado no presente estudo.

Abrir-se à possibilidade de falar sobre a R/E com o paciente, desmistificando a imposição de valores $\mathrm{e}$ crenças, permitindo que essa temática seja abordada com respeito é corporificar o cuidado integral e humanizado. A negação da R/E pressupõe a falta do cuidado integral, deixando à margem a dimensão espiritual do ser humano, que em momentos de dor e sofrimento aparece com grande relevância. Assim, o cuidado de Enfermagem deve estar não apenas atento a essa realidade, como também aberto a revisões em suas práticas e protocolos, em diálogo constante com as demandas religiosas-espirituais que, porventura, emerjam na linha de cuidado.

\section{CONSIDERAÇÕES FINAIS}

Ao se apropriar da temática da R/E o enfermeiro conhece subsídios e aportes teóricos para nortear a aplicação de intervenções espirituais no processo de tomada de decisão para a condução da assistência. As ações em saúde propostas pela NIC podem ser norteadoras de como a dimensão da R/E pode, de fato, ser corporificada no cuidado. $O$ emprego desses protocolos pode disparar reflexões importantes não apenas para os profissionais em atuação, mas também para a formação em Enfermagem, de modo que os currículos de cursos de graduação e de pós-graduação possam corporificar essas orientações e promover debates que diminuam as incertezas quanto a consideração dessa dimensão na atenção em saúde, campo este que, inequivocamente, tem recebido importantes contribuições da ciência do cuidado. Sugere-se, portanto, estudos futuros necessários para mensurar a importância da assistência espiritual, possivelmente avaliando as ações de cuidado espiritual cotejadas pela NIC, o que pode gerar incrementos para a discussão da R/E na saúde de modo ampliado e baseado em evidências científicas. 


\section{REFERÊNCIAS}

1. AlmeidA AM, LUCChetTI G. Panorama das Pesquisas em Ciência, Saúde e Espiritualidade. Cienc. Cult. 2016; 68(1).

2. BORIS GD, et al. Influência da Fenomenologia e Existencialismo na Gestalt-Terapia. Estudos de Psicologia Campinas, 34, 2017.

3. BULECHEK GM, et al. NIC - Classificação das Intervenções de Enfermagem. 6 ? $^{\mathbf{a}}$ ed. Tradução de Denise Costa Rodrigues. Rio de Janeiro: Elsevier; 2016.

4. CALDEIRA S, et al. Clinical validation of the nursing diagnosis spiritual distress in câncer patients nundergoing chemotherapy. International Journal of Nursing Knowledge, 2016.

5. CAMPOS AF, RIBEIRO JP. Psicoterapia e espiritualidade: da gestaltterapia à pesquisa contemporânea. Rev abordagem gestalt [Internet]. 2017; 23(2):211-8.

6. CARLSON LE, et al. Randomized-controlled trial of mindfulness-based cancer recovery versus supportive expressive group therapy among distressed breast cancer survivors (MINDSET): long-term follow-up results. Psychooncology. 2016; 25(7):750-9.

7. CARVALHO CC, et al. Precesses intercessórias: efeito na recuperação da saúde das pessoas em tratamento médico. Índice Enferm, Granada, 2013; 22(3).

8. CHEUNG KC, et al. Buddhist Counseling: Implications for Mental Health Professionals. Spiritual Clin Pract . 2017; $4(2): 113-28$.

9. CUNHA VF, SCORSOLINI-COMIN F. Best professional practices when approaching religiosity/spirituality in psychotherapy in Brazil. Couns Psychoth Res. 2019.

10. FREIRE MEM, et al.. Spiritual and religious assistance to cancer patients in the hospital context. J Res Fundam Care Online, 2017.

11. JANG JE. The Interactive Play and a Persuasive God: A Psychoanalytic Approach to Re-envisioning Pastoral Care and Counseling. J Pastor Care Couns [Internet]. 2016.

12. KOENIG HG. Religião, espiritualidade e psiquiatria: uma nova era na atenção à saúde mental. Rev Psiq Clín. 2007.

13. MENDONÇA AG et al. Aconselhamento e assistência espiritual a pacientes em quimioterapia: uma reflexão à luz da Teoria de Jean. Esc Anna Nery 2018.

14. MILLER AJ. The Spiral Staircase: A Narrative Approach to Pastoral Conversation. J Pastor Care Couns. 2016.

15. NEELY D, MINFORD EJ. Current status of teaching on Spirituality in UK medical Schools. Med Educ, 42 (2), 2008.

16. PEDRÃO RB, BERESIN R. O enfermeiro frente à questão da espiritualidade. Einstein, São Paulo, 2010.

17. ROBITSCHEK C, HARDIN EE. The future of counseling psychology researchviewed through the cultural lens approach. J Couns Psychol, 2017.

18. SCORSOLINI-COMIN F. Elementos do aconselhamento multicultural aplicados à psicoterapia em contexto etnopsicológico. Estud PesquiPsicol [Internet].,2015.

19. SIMÕES ND, et al. Espiritualidade e saúde: experiência de uma disciplina na graduação de enfermagem. Revista de Enfermagem da UFSM, [S.I.], 2018; 8(1): 181-191.

20. SOUSA FFPR, et al. Religious/Spiritual Coping by People with Cancer Undergoing Chemotherapy: Integrative Literature Review. SMAD Ver Eletr Saúde Ment Álcool Drog [Internet]. 2017. 